\title{
House Dust Mites in Brazil - An Annotated Bibliography
}

\section{Raquel S Binotti/ ${ }^{+}$, João RO Muniz*, Ilma A Paschoal*, Angelo P do Prado, Celso H Oliveira*}

\author{
Departamento de Parasitologia, Instituto de Biologia *Departamento de Clínica Médica, Faculdade de Medicina, \\ Universidade Estadual de Campinas, Caixa Postal 6111, 13081-970 Campinas, SP, Brasil
}

House dust mites have been reported to be the most important allergen in human dwellings. Several articles had already shown the presence of different mite species at homes in Brazil, being Pyroglyphidae, Glycyphagidae and Cheyletidae the most important families found. This paper is an annotated bibliography that will lead to a better knowledge of house dust mite fauna in Brazil.

Key words: allergy - atopy - house dust mites - Dermatophagoides - Blomia - Brazil

Allergies of the respiratory tract have been considered one of the principal infirmities of modern man, provoked mainly by immediate sensitiveness to various indoor dust aeroallergens. Outstanding among these are the allergens derived from house dust mites belonging to different genera and species, mainly Dermatophagoides pteronyssinus, $D$. farinae and Euroglyphus maynei (Platts-Mills \& Chapman 1987).

There is a great variation in the acarine fauna among the different regions of the world. The diversity of mite fauna of a given (micro)habitat is not only due to direct influence of environmental temperature and humidity upon the development of mites (Wharton 1976, Bronswijk 1981). Additional ecological factors and evolutionary stochalistic factors may also play a role in mite diversity. This study presents a detailed bibliography of the house dust mite in Brazil. A great variety of species has been observed, the most outstanding of which are those from genera of the families Pyroglyphidae (Dermatophagoides and Euroglyphus), Glycyphagidae (Blomia), Cheyletidae (Cheyletus) as well as Acaridae (Tyrophagus).

The objective of the present paper was to draw up a bibliographic survey of the main studies on the presence of mites in house dust in several parts of the country.

\section{BIBLIOGRAPHIC SURVEY}

A total of 101 works (books, articles and abstracts of congresses and symposia) already published on mites in house dust in Brazil were included.

\footnotetext{
${ }^{+}$Corresponding author. Fax: +55-19-3233.5319. E-mail: raquelbinotti@uol.com.br Received 17 January 2001 Accepted 16 July 2001
}

The result of this bibliographic survey showed the presence of a large number of mites belonging to a total of 23 superfamilies, 28 families, 40 genera and 39 species, the acarine genera of the families Pyroglyphidae, Glycyphagidae, Cheyletidae and Acaridae being the most frequently mentioned. Other families reported were Tarsonemidae, Chortoglyphidae, Ascidae and Tydeidae.

Frequent reference to the genus Dermatophagoides (Pyroglyphidae) was registered. The most cited species were $D$. pteronyssinus and $D$. farinae (Tables I, III). The prevalence reported ranged from 3.7 to $89.3 \%$ (Rosa 1978, Baggio et al. 1992a) and 0.05 to $39.2 \%$ (Moreira 1975, Baggio \& Ambrózio 1992b) respectively, the mite $D$. pteronyssinus being found in the entire national territory and mentioned in 69 articles. The species $D$. farinae was reported in almost all of the country and was cited in 28 articles. Other genera of this family were Euroglyphus, E. maynei being the principal species (prevalence of 0.2 to 6.1\%) (Rosa 1978, Baggio et al. 1988a); Pyroglyphus (P. africanus 0.6 to $2.5 \%$ ) (Rosa 1978, Baggio et al. 1988a) and Sturnophagoides (S. brasiliensis) (Galvão \& Guitton 1986a) (Tables II, III). The family Glycyphagidae was the second most frequently cited (61 articles), genera Blomia (B. tropicalis 3.8 to $79.5 \%$ ) (Rosa 1978, Chagas et al. 2000b) (Tables I, III) and Glycyphagus (G. domesticus - 6.8 to $36.5 \%$ ) (Moreira 1975, Baggio et al. 1987), being the principal mites of this family. The family Cheyletidae was also frequently cited, genus Cheyletus (0.1 to 17.6\%) (Baggio \& Ambrózio 1992a, Baggio et al. 1992a) and the species $C$. malaccensis (1.5 to 18.2\%) (Bonini et al. 1988, Bernd et al. 1990) being the most reported. Reference to the species Tyrophagus putrescentiae (Acaridae) was observed in 28 articles, with a prevalence varying between 0.2 to $10.5 \%$ (Rosa 1978, Jorge Neto 1984). Besides, frequent reference was noted re- 
TABLE I

Principal house dust mites in Brazil: references and prevalence reported

\begin{tabular}{|c|c|c|c|c|c|c|c|c|c|}
\hline \multirow[b]{2}{*}{ Name } & \multirow[b]{2}{*}{$\mathrm{N}$} & \multicolumn{3}{|c|}{ Minimum prevalence } & \multirow[b]{2}{*}{ Locality } & \multicolumn{4}{|c|}{ Maximum prevalence } \\
\hline & & $\%(-)$ & Year & Reference & & $\%(+)$ & Year & Reference & Locality \\
\hline Dermatophagoides pteronyssinus & 69 & 3.7 & 1978 & Rosa & Rio Claro/SP & 89.3 & $1992 \mathrm{a}$ & Baggio et al. & Catanduva/SP \\
\hline Dermatophagoides farinae & 28 & 0.05 & $1992 b$ & Baggio \& Ambrózio & Brasil & 39.2 & 1975 & Moreira & Belo Horizonte/MG \\
\hline Euroglyphus maynei & 23 & 0.2 & 1978 & Rosa & Rio Claro/SP & 6.1 & $1988 \mathrm{a}$ & Baggio et al. & Luis Antônio/SP \\
\hline Pyroglyphus africanus & 8 & 0.6 & $1988 \mathrm{a}$ & Baggio et al. & Luis Antônio/SP & 2.5 & 1978 & Rosa & Rio Claro/SP \\
\hline Tyrophagus putrescentiae & 28 & 0.2 & 1978 & Rosa & Rio Claro/SP & 10.5 & 1984 & Jorge Neto & São Paulo/SP $c$ \\
\hline Blomia tropicalis & 61 & 3.8 & $2000 b$ & Chagas et al. & Araguaína/TO & 79.5 & 1978 & Rosa & Rio Claro/SP \\
\hline Glycyphagus domesticus & 4 & 6.8 & 1987 & Baggio et al. & São Paulo/SP ${ }^{a}$ & 36.5 & 1975 & Moreira & Belo Horizonte/MG ${ }^{d}$ \\
\hline Chortoglyphus arcuatus & 17 & 0.8 & 1988 & Baggio \& Croce & São Paulo/SP $b$ & 18.7 & 1984 & Jorge Neto & São Paulo/SP \\
\hline Cheyletus sp. & 7 & 0.1 & $1992 \mathrm{a}$ & Baggio \& Ambrózio & Castanhal/PA & 17.6 & $1992 \mathrm{a}$ & Baggio at al. & Araraquara/SP \\
\hline Cheyletus eruditus & 2 & 0.6 & $1988 \mathrm{a}$ & Baggio et al. & Luis Antônio/SP & 6.6 & 1975 & Moreira & Belo Horizonte/MG \\
\hline Cheyletus fortis & 5 & 0.6 & $1988 \mathrm{a}$ & Baggio et al. & Luis Antônio/SP & 0.9 & 1988 & Mello et al. & $\begin{array}{l}\text { Belém, Icoaracy and } \\
\text { Castanhal/PA }\end{array}$ \\
\hline Cheyletus malaccensis & 35 & 1.5 & 1990 & Bernd et al. & Porto Alegre/RS & 18.2 & 1988 & Bonini et al. & Araraquara/SP \\
\hline Tarsonemus sp. & 19 & 0.5 & $1988 \mathrm{a}$ & Croce et al. & São Paulo/SP & 66.7 & $2000 \mathrm{a}$ & Chagas et al. & Campinas/SP ${ }^{e}$ \\
\hline Spinibdella cronini & 4 & 0.3 & 1978 & Rosa & Rio Claro/SP & 4.5 & 1988 & Bonini et al. & Araraquara/SP \\
\hline Blattisocius tarsalis & 3 & 1.2 & 1990 & Baggio et al. & Belém/PA & 1.2 & $1988 \mathrm{a}$ & Baggio et al. & Luis Antônio/SP \\
\hline Suidasia pontifica & 15 & 0.3 & $1999 b$ & Binotti et al. & Bragança Paulista/SP & 1.1 & 1999 & Oliveira & Campinas/SP \\
\hline Aleuroglyphus ovatus & 8 & 1.6 & 1988 & Baggio \& Croce & São Paulo/SP $b$ & 17.5 & 1987 & Baggio et al. & São Paulo/SP $a$ \\
\hline Demodex sp. & 2 & 0.3 & $1999 b$ & Binotti et al. & Bragança Paulista/SP & 0.6 & 1999 & Oliveira & Campinas/SP \\
\hline Demodex foliculorum & 3 & 0.8 & 1980 & Croce et al. & São Paulo/SP $b$ & 4.9 & 1988 & Baggio \& Croce & São Paulo/SP $b$ \\
\hline Sarcoptes scabiei & 2 & 3.1 & 1980 & Croce et al. & São Paulo/SP $b$ & 9.8 & 1988 & Baggio \& Croce & São Paulo/SP $b$ \\
\hline
\end{tabular}

$\mathrm{N}$ : number of articles; $a$ : cereal samples; $b$ : samples of human skin shavings; $c$ : T. putrescentiae also in sweets (98.9\%; Franzolin et al. 1994), in cereals (48\%; Baggio et al. 1987) and pantries (46.4\%; Binotti et al. 1999a); $d$ : 11.4\% if corrected, according to Rosa and Flechtmann (1979), being them 36\% as maximum porcentage in Moreira (1978). Authors' note: the species B. tropicalis was described only in 1973 (Bronswijk et al.); $e$ : samples in dust of tatami mattresses; \% (-): smallest percentage cited; \% (+): largest percentage cited 
TABLE II

Little-mentioned house dust mites in Brazil

\begin{tabular}{|c|c|c|c|c|c|}
\hline Name & $\mathrm{N}$ & $\%$ & Year & Reference & Locality \\
\hline Dermatophagoides neotropicalis ${ }^{a}$ & 2 & ? & 1986b & Galvão \& Guitton & Brasil \\
\hline Dermatophagoides microceras & 1 & $?$ & 1989 & Baggio et al. & São Paulo/SP \\
\hline Euroglyphus longior & 1 & 0.6 & $1988 \mathrm{a}$ & Baggio et al. & Luis Antônio/SP \\
\hline Sturnophagoides brasiliensis & 1 & $?$ & $1986 a$ & Galvão \& Guitton & Brasil \\
\hline Lepidoglyphus sp. & 4 & 0.04 & 1978 & Rosa & Itaguaí/SC, Rio Claro/SP \\
\hline Gohieria sp. & 3 & 0.6 & 1999 & Oliveira & Campinas/SP \\
\hline Austroglycyphagus lukoschusi & 3 & 0.04 & 1978 & Rosa & Brasil \\
\hline Lepidoglyphus destructor & 2 & 4 & 1999 & Serravale \& Medeiros & Salvador/BA \\
\hline Blomia kulagini & 1 & $?$ & $1988 b$ & Croce et al. & Brasil \\
\hline Acarus siro & 2 & $?$ & 1986 & Flechtmann & Brasil \\
\hline Suidasia nesbitti & 1 & 2.1 & $1999 a$ & Binotti et al. & Campinas/SP ${ }^{b}$ \\
\hline Caloglyphus berlesei & 1 & 4.9 & 1988 & Baggio \& Croce & São Paulo/SP ${ }^{c}$ \\
\hline Thyreophagus entomophagus & 1 & $?$ & 2000 & Mazzuco et al. & Florianópolis/SC ${ }^{d}$ \\
\hline Histiostoma $\mathrm{sp}$. & 1 & 1.2 & 1987 & Baggio et al. & São Paulo/SP $e$ \\
\hline Trouessartia sp. & 1 & 0.8 & 1980 & Croce et al. & São Paulo/SP ${ }^{c}$ \\
\hline Tarsonemus granarius & 2 & 3.0 & 1988 & Garcia et al. & Macapá/AP \\
\hline Tarsonemus fusaeri & 1 & 29.6 & $1988 \mathrm{c}$ & Croce et al. & São Paulo/SP $f$ \\
\hline Ker bakeri & 2 & 0.6 & 1978 & Rosa & Rio Claro/SP \\
\hline Grallacheles bakeri & 2 & 0.1 & 1978 & Rosa & Rio Claro/SP \\
\hline Cheletonella caucasica & 2 & $?$ & $2000 \mathrm{a}$ & Ezequiel et al. & Juiz de Fora/MG \\
\hline Cheletonella vespertillionis & 1 & $?$ & $2000 \mathrm{a}$ & Ezequiel et al. & Juiz de Fora/MG \\
\hline Cheyletus backeri & 1 & $?$ & $1988 \mathrm{c}$ & Baggio et al. & Cascavel/PR \\
\hline Demodex brevis & 1 & $?$ & 1993 & Madeira \& Sogayar & Botucatu/SP ${ }^{c}$ \\
\hline Pyemotes sp. & 1 & 31.5 & $1998 b$ & Oliveira et al. & Campinas/SP ${ }^{g}$ \\
\hline Tydeus sp. & 4 & 7.5 & $1988 \mathrm{c}$ & Croce et al. & São Paulo/SP $f$ \\
\hline Parapronematus sp. & 2 & 0.2 & 1978 & Rosa & Rio Claro/SP \\
\hline Paralorryia sp. & 2 & 0.2 & 1978 & Rosa & Rio Claro/SP \\
\hline Raphygnathus sp. & 2 & 0.04 & 1978 & Rosa & Rio Claro/SP \\
\hline $\begin{array}{l}\text { Kleemannia sp. } \\
\text { Phytoseius sp. }\end{array}$ & $\begin{array}{l}1 \\
1\end{array}$ & $\begin{array}{c}0.5 \\
?\end{array}$ & $\begin{array}{l}1999 a \\
1984\end{array}$ & $\begin{array}{l}\text { Binotti et al. } \\
\text { Jorge Neto }\end{array}$ & $\begin{array}{l}\text { Campinas/SP } b \\
\text { São Paulo/SP }\end{array}$ \\
\hline Blattisocius dendriticus & 4 & 0.1 & 1978 & Rosa & Rio Claro/SP \\
\hline Blattisocius keegani & 3 & 0.1 & 1978 & Rosa & Rio Claro/SP \\
\hline Blattisocius sp. & 2 & 0.3 & 1992 & Bernd et al. & Porto Alegre/RS \\
\hline Fuscuropoda sp. & 1 & 0.6 & $1988 \mathrm{a}$ & Baggio et al. & Luis Antônio/SP \\
\hline Haplochthonius simplex & 2 & 0.4 & 1978 & Rosa & Rio Claro/SP \\
\hline Haplochthonius sp. & 1 & 0.6 & 1975 & Moreira & Belo Horizonte/MG \\
\hline Podoribates sp. & 2 & 0.04 & 1978 & Rosa & Rio Claro/SP \\
\hline Scheloribates sp. & 2 & 0.9 & 1978 & Rosa & Rio Claro/SP \\
\hline Mochlozetes sp. & 2 & 0.5 & 1978 & Rosa & Rio Claro/SP \\
\hline Belba sp. & 1 & 0.9 & 1975 & Moreira & Belo Horizonte/MG \\
\hline
\end{tabular}

The localities cited above were from the first article to cite each mite species. $a$ : the former name (D. deane $i)$ has been synonymized; N: total number of articles related to the acarine species reported; $b$ : samplings of dust in pantries; $c$ : scale samples of human skin; $d$ : samplings of wheat flour; $e$ : cereal samples; $f$ : samplings of dust in libraries; $g$ : dust samplings of central files 
1180 House Dust Mites in Brazil • Raquel S Binotti et al.

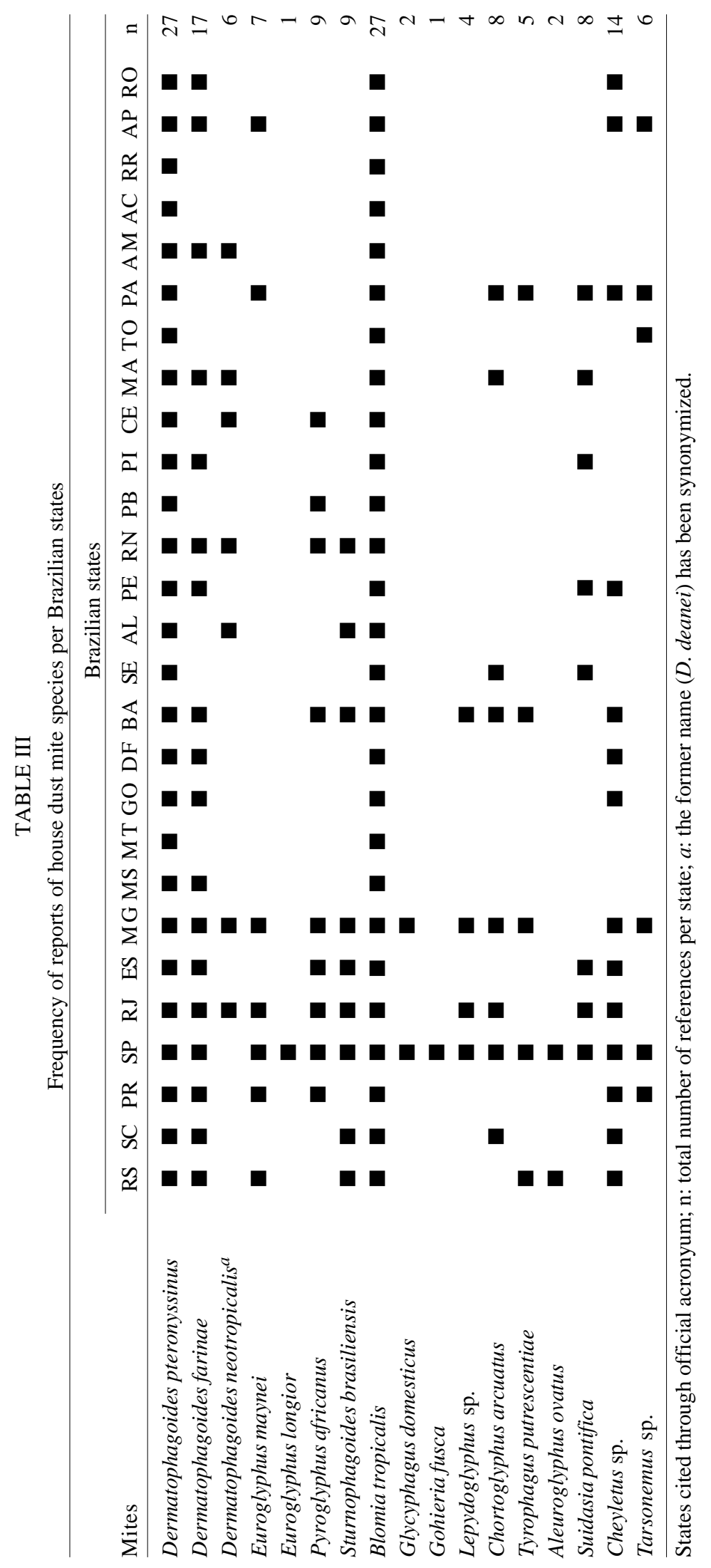


garding families Chortoglyphidae (Chortoglyphus arcuatus - 0.8 to $18.7 \%$ ) (Jorge Neto 1984, Baggio $\&$ Croce 1988) and Tarsonemidae (Tarsonemus sp. - 0.5 to 66.7 - in tatami mattresses) (Croce et al. 1988a, Chagas et al. 2000a). Other genera and species of mites were referred to in a few articles, these being only rarely found in samplings of Brazilian house dust (Table II). The results of this survey have therefore shown that the mites of the families Pyroglyphidae, Glycyphagidae and Cheyletidae are the most cited in the published papers regarding the acarine fauna of house dust in Brazil.

Brazil is a tropical country with relative humidity and average annual temperature around $70 \%$ and $27^{\circ} \mathrm{C}$, respectively. Such situation is strongly favorable to the development of house mites (Bronswijk 1981, Flechtmann 1986).

Part of the mites found have already been identified as sensitizing agents of humans, as there are studies demonstrating immediate cutaneous sensitivity to specific extracts from the majority of these arachnids, including Cheyletus malaccensis e Tarsonemus sp. (Korsgaard \& Hallas 1979, Ambrózio et al. 1989, Oliveira et al. 1996).

Other acarine species were rarely cited, to the point where in several cases they may be considered as "contaminants", i.e., originating from an environment other than that of the home, such as gardens, in the case of phytophagus mites of the families Eriophyidae and Tetranychidae. The allergenic potential of these acarine species is unknown to the present date.

Lastly, it is outstanding that the majority of studies refer to localities in the South and above all Southeast region, there being few references regarding samplings of acarine fauna of the other Brazilian regions. It is advisable therefore to encourage studies that may go ahead with the evaluation of the acarine fauna in the various regions of Brazil, attempting to establish more detailed relations about their interaction with the human being and regional characteristics of populational habits and the home environment.

We may conclude from this bibliographical revision that the most common species of house dust mites found in Brazil were D. pteronyssinus, $B$. tropicalis and C. malaccensis. Other species/genera also frequently reported in the articles were $D$. farinae, Euroglyphus (Pyroglyphidae), Tyrophagus (Acaridae), Chortoglyphus (Chortoglyphidae) and Tarsonemus (Tarsonemidae).

\section{ANNOTATED BIBLIOGRAPHY}

Amaral V 1967. Nota prévia sobre a ocorrência de Dermatophagoides pteronyssinus (Trouessart, 1897), em São Paulo (Psoroptidae: Sarcoptiformes). Scientific Meeting, Sociedade Paulista de Medicina
Veterinária, 26 May 1967.

Amaral V 1968. Sobre a ocorrência do ácaro Dermatophagoides pteronyssinus (Trouessart, 1897) no Brasil (Psoroptidae: Sarcoptiformes). Rev Med Vet São Paulo 3: 296-300.

Ambrózio LC, Baggio D, Mori JC, Fernandes MFM, Mello JF 1988. Suidasia pontificia - alergizante das vias aéreas? XXI Congresso Brasileiro de Alergia e Imunopatologia e IV Congresso de Alergia e Imunologia do Cone Sul, Florianópolis, SC, Oswaldo Seabra Prize.

Ambrózio LC, Baggio D, Mori JC, Fernandes MFM, Kase MT, Mello JF 1989. Suidasia pontificia: alergizante de vias respiratórias? Investigação preliminar de antígenos de outros gêneros de ácaros da poeira domiciliar. Rev Bras Alerg Imunopatol 12: $15-23$.

Antilla MA, Baggio D, Croce J 1987. Estudo comparativo de qualificação de ácaros de poeira domiciliar em casas com e sem carpete e a relação deste tipo de piso com alergias respiratórias no homem. Cong Bras de Alerg Imunol, Rio de Janeiro, RJ, p. 17.

Antila M, Nali M, Abdala N, Baggio D, Croce J 1988. Ácaros do pó domiciliar de Mogi das Cruzes, SP. Rev Bras Alerg Imunopatol 11: A119.

Antunes HBB, Bernd LAG 1994. Comparação da acarofauna no piso e objetos da cama. Rev Bras Alerg Imunopatol 17: A073.

Antunes HBB, Di Gesu G, Di Gesu RW, Bernd LAG 1994. Exposição a ácaros em moradias da cidade de Rio Grande (RS). Rev Bras Alerg Imunopatol 17: A072.

Arruda LK, Rizzo MC, Chapman MD, FernandezCaldas E, Baggio D, Platts-Mills TAE, Naspitz CK 1991. Exposure and sensitization to dust mite allergens among asthmatic children in São Paulo, Brazil. Clin Exp Allergy 21: 433-439.

Baduv AC, Ferrari FP, Rosário NA, Kuvalhuk LS 1996. Pesquisa de ácaros em amostras de farinha de trigo comercializadas em Curitiba. Rev Bras Alerg Imunopatol 19: 57.

Baggio D, Ambrózio LC 1992a. Domestic mites in the Brazilian countries. Rev Bras Alerg Imunopatol 15: A93.

Baggio D, Ambrózio LC 1992b. Household mites from Brazil summary and annual seazonal variation. Rev Bras Alerg Imunopatol 15: A91.

Baggio D, Croce J 1986. Ácaros encontrados associados a dermatites atópicas no homem. Resumos do $1^{\circ}$ Seminário sobre Vetores Urbanos e Animais Sinantrópicos, São Paulo, SP, p. 32.

Baggio D, Croce J 1988. Encontro de ácaros associados a dermatites. Rev Bras Alerg Imunopatol 11: A116.

Baggio D, Ambrózio LC, Antilla MA 1989. Ácaros ambientais e as manifestações alérgicas. Rev Bras Alerg Imunopatol 12: 56-68.

Baggio D, Ambrózio LC, Antilla MA 1992a. Mites in house dust in cities from the state of São Paulo studies of seasonability in population. Rev Bras Alerg Imunopatol 15: A92.

Baggio D, Ambrózio LC, Cordaro C 1992b. Household mites from South American Countries - A review. 
Rev Bras Alerg Imunopatol 15: A90.

Baggio D, Croce J, Castro FM 1988b. Ácaros do pó domiciliar de Cubatão, S. Paulo. Rev Bras Alerg Imunopatol 11: A118.

Baggio D, Machado JLC, Croce J 1988c. Variação sazonal de ácaros do pó em Cascavel. Rev Bras Alerg Imunopatol 11: A121.

Baggio D, Bellesi N, Ambrósio LC, Cretelli NBS 1990. Ácaros do pó domiciliar na cidade de Belém, Pará (Brasil). Rev Bras Alerg Imunopatol 13: A74.

Baggio D, Croce J, Cruz-Jovane M, Jordan MAP 1985. Contribuição para o conhecimento da fauna acarológica de poeira domiciliar da cidade de São Paulo. Resumos do XII Cong Bras de Zoologia, Campinas, SP, p. A82.

Baggio D, Croce J, Zuppi L, Mello W 1988a. Ácaros do pó domiciliar em Luiz Antônio, SP. Rev Bras Alerg Imunopatol 11: A120.

Baggio D, Figueiredo SM, Flechtmann CHW, Zambon GQ, Miranda SHG 1987. Avaliação da presença de ácaros em cereais armazenados na grande São Paulo. Resumos do XI Cong Bras de Entomologia, Vol. 1, Campinas, SP, p. 21.

Bernd LAG, Baggio D, Cordaro C 1990. Ácaros do pó domiciliar da cidade de Porto Alegre, RS - Brasil. Rev Bras Alerg Imunopatol 13: A140.

Bernd LAG, Baggio D, Becker AB, Ambrózio LC 1992. Variação sazonal anual da acarofauna em Porto Alegre (RS). Rev Bras Alerg Imunopatol 15: A80.

Bernd LA, Baggio D, Becker AB, Ambrózio LC 1994. Identificação e estudo da atividade sensibilizante de ácaros domésticos em Porto Alegre (RS). Rev Bras Alerg Imunopatol 17: 23-33.

Binotti RS, Daolio A, Oliveira CH, Muniz, JRO, Prado AP 2000a. Fauna acarina da poeira de colchões na cidade de Pouso Alegre - MG. Rev Bras Alerg Imunopatol 23: 196.

Binotti RS, Muniz JRO, Oliveira CH, Prado AP 2000c. Levantamento de ácaros em poeira de sofás. Rev Bras Alerg Imunopatol 23: 209.

Binotti RS, Muniz JRO, Oliveira CH, Prado AP 2000d. Levantamento de ácaros em poeira de diferentes locais em 58 residências de Campinas/SP. Rev Bras Alerg Imunopatol 23: 189.

Binotti RS, Oliveira CH, Muniz JRO, Prado AP 1999a. Fauna acarina em amostras de poeira de dispensas domiciliares em Campinas - SP. IV Congresso Aberto aos Estudantes de Biologia, Unicamp, SP, Abstracts Book 1.01, p. 38.

Binotti RS, Oliveira CH, Muniz JRO, Pinho Jr AJ, Prado AP 1999b. Fauna acarina da poeira intradomiciliar na cidade de Bragança Paulista - SP. IV Congresso Aberto aos Estudantes de Biologia, Unicamp, SP, Abstracts Book 2.31, p. 147.

Binotti RS, Oliveira CH, Muniz JRO, Prado AP 2000b. Levantamento da fauna acarina em amostras de poeira de cortinas na cidade de Campinas - SP. Rev Bras Alerg Imunopatol 23: 209.

Bonini, E, Croce J, Baggio D 1988. Ácaros do pó domiciliar de Araraquara, SP. Rev Bras Alerg Imunopatol 11: A107.

Bronswijk JEMH 1981. House Dust Biology: for Aller- gists, Acarologists and Mycologists, NIB Publishers, Zoelmond, The Netherlands, 316 pp.

Cardoso RRA, Barbosa CAA, Nascimento JJ 1979. Dust mites. Allergy 34: 257-260.

Castro FFM, Mori JC, Cruz Filho AA, Melo Jr JF, Baiocchi Jr G, Reis EAPR, Bernd LAG, Emerson MF, Fernandes MFM, Croce J 1995. Rinite e alérgenos intra e extra-domiciliares. Rev Bras Alerg Imunopatol 18: 178-184.

Chagas KN, Binotti RS, Oliveira CH, Muniz JRO, Prado AP 2000a. Avaliação da fauna acarina em amostras de poeira de 'tatames' presentes em academias de judô. Rev Bras Alerg Imunopatol 23: 210.

Chagas KN, Muniz JRO, Binotti RS, Oliveira CH, Chagas KDN, Prado AP 2000b. Primeiro levantamento de ácaros em poeira de casas da cidade de Araguaína - Tocantins. Rev Bras Alerg Imunopatol 23: 209.

II Consenso Brasileiro no Manejo da Asma 1998. Rev Bras Alerg Imunopatol 21 (Supl. 1): 171-276.

Corral JNE 1997. Ácaros, Laboratório de Pesquisa da Disciplina de Gastroenterologia Pediátrica, Escola Paulista de Medicina, Universidade Federal de São Paulo, São Paulo, 20 pp.

Croce J, Baggio D, Castro FFM 1986. Estudos preliminares sobre a fauna acarológica da poeira domiciliar no município de Cubatão, SP. XX Congr Bras Alerg e Imunopatol, Rio de Janeiro, RJ, p. 10.

Croce J, Baggio D, Garcia LRF 1987. The epidemiology of bronchial asthma in the Brazilian Amazonia. V Congresso Luso-Brasileiro de Alergia e Imunopatologia, Lisboa, Portugal, p. 32.

Croce J, Baggio D, Jorge Neto J 1988a. Seasonal changes of the house dust species in the city of São Paulo Brasil. NER Allergyn Proc 9: 342.

Croce J, Baggio D, Zuppi LJ, Alario MCT 1980. Presença de ácaros em pacientes com dermatoses. Rev Bras Alerg Imunopatol 2: 192-193.

Croce J, Baggio D, Riva G, Centanni G 1988b. An immunological study on the mite Blomia kulagini in Brazilian patients. Rev Bras Alerg Imunopatol 11: A111.

Croce M, Manso ERC, Sales JM, Baggio D, Croce J, Diniz M, Marques MC 1988c. Ácaros do pó de bibliotecas da Universidade de São Paulo. Rev Bras Alerg Imunopatol 11: A117.

Ezequiel O, Gazêta G, Amorim M, Serra-Freire N 2000a. Avaliação qualitativa da acarofauna do ecossistema domiciliar na cidade de Juiz de Fora, Estado de Minas Gerais, Brasil. Rev Bras Alerg Imunopatol 23: 188.

Ezequiel O, Gazêta G, Serra-Freire N 2000b. Caso reportado de dermatite por ácaros da família Cheyletidae. Rev Bras Alerg Imunopatol 23: 205.

Ezequiel O, Oliveira G, Barros R, Gazêta G, Serra-Freire N 2000c. Infestação por ácaros no laboratório de patologia do HU-UFJF: avaliação das espécies de ácaros e seu possível papel na vetoração de agentes. Rev Bras Alerg Imunopatol 23: 188.

Flechtmann CHW 1972. Ácaros de Importância Agrícola, Nobel, São Paulo, 150 pp.

Flechtmann CHW 1973. Ácaros de Importância Médicoveterinária, Nobel, São Paulo, 192 pp.

Flechtmann CHW 1986. Ácaros em Produtos Arma- 
zenados e na Poeira Domiciliar, Escola Superior de Agricultura 'Luiz de Queiróz', Departamento de Zoologia, Universidade de São Paulo, 97 pp.

Flechtmann CHW, Rosa AE 1980. Estudo sobre a fauna acarina de poeira domiciliar no Brasil. Rev Bras Alerg Imunopatol 2: 91-94.

Flechtmann CHW, Costa CP, Maielli JA 1998. A Residência para o Alérgico - Construção e Adaptação, Unimep, Piracicaba, SP, 57 pp.

Franzolin MR, Baggio D, Correia M, Rodrigues RMMS 1994. Presença de ácaros em doces de amendoim e de leite vendidos por ambulantes na cidade de São Paulo. Rev Inst Adolfo Lutz 54: 11-15.

Galvão AB, Guitton N 1986a. Ácaros em poeira domiciliar das capitais brasileiras e ilha de Fernando de Noronha. Mem Inst Oswaldo Cruz 81: 417-430.

Galvão AB, Guitton N 1986b. Dermatophagoides deanei sp. n., nova espécie de ácaro piroglifídeo encontrada no Brasil em poeira domiciliar. Mem Inst Oswaldo Cruz 81: 241-244.

Garcia LRF, Baggio D, Croce J, Espírito Santo MNA 1988. Ácaros do pó domiciliar em Macapá (AP). Rev Bras Alerg Imunopatol 11: A108.

Geller M 1996. Alergia aos ácaros no Rio de Janeiro. Análise prospectiva em 700 pacientes com asma e(ou) rinite. $J B M 71: 164-170$.

Geller M, Esch RE, Fernandez-Caldas E 1995. Domestic mite allergic sensitivity in Rio de Janeiro. Rev Bras Alerg Imunopatol 18: 13-18.

Gomes VB, Costa CP, Rosa AE, Flechtmann CHW 1980a. Observações sobre a prevalência de ácaros em residências de alérgicos na cidade de Piracicaba São Paulo. Simpósio de Ácaros e Alergia, Sociedade Brasileira de Alergia e Imunopatologia - regional São Paulo, São Paulo, SP.

Gomes VB, Costa CP, Rosa AE, Flechtmann CHW 1980b. Observações sobre a prevalência de ácaros em residências de alérgicos na cidade de Piracicaba São Paulo. Rev Bras Alerg Imunopatol 2: 95-98.

Greco DB, Moreira NS, Filogonio JBC, Greco JB 1974. Demonstração da presença de ácaros em pó domiciliar de Belo Horizonte e outras cidades de Minas Gerais. Resumo do XIV Congresso de Alergia e Imunopatologia, October; Recife, PE, p. 1.

Goudoris ES, Baggio D, Ambrózio LC, Aires VT, Prado EA 1994. Estudos dos extratos de ácaros mais adequados para os testes cutâneos no IPPMG. Rev Bras Alerg Imunopatol 17: A071.

Jorge Neto J 1984. Contribuição para o Estudo da Fauna Acarina da Poeira Domiciliar em Habitações da Cidade de São Paulo, MSc Thesis, Faculdade de Medicina, Universidade de São Paulo.

Jorge Neto J, Croce J, Baggio D 1980. Ácaros da poeira domiciliar na cidade de São Paulo. Rev Bras Alerg Imunopatol 2: 140.

Jorge Neto J, Baggio D 1982. Inquérito sobre a fauna acarológica da poeira domiciliar na cidade de São Paulo, SP. Resumos do VII Cong Bras Parasitol, Porto Alegre, RS, p. 91.

Jorge Neto J, Baggio D, Croce J, Levy LH 1984. Ácaros da poeira domiciliar: estudo em habitações de São Paulo. XIX Cong Bras de Alerg e Imunopatol e IV
Cong Luso-brasil de Alergia e Imunopatol; Olinda, PE, p. 88 .

Jorge Neto J, Baggio D, Croce J, Levy LH 1985. Ácaros de poeira domiciliar - estudo em habitações da cidade de São Paulo. Resumos do XII Cong Brasil de Zoologia, Campinas, SP, p. A83.

Jota VCS, Holanda ACA, Régis LDRB 1993a. Levantamento da acarofauna em poeira domiciliar na região metropolitana do Recife-PE. Anais do XIV Congr Brasil Entomologia, Piracicaba, SP, p. 730.

Jota VCS, Holanda ACA, Régis LDRB 1993b. Análise faunística da acarofauna em poeira domiciliar na região metropolitana do Recife-PE. Anais do XIV Congr Brasil Entomologia, Piracicaba, SP, p. 731.

Jovane MC, Jordan MAP, Baggio D, Croce J 1984. Contribuição para o conhecimento da fauna acarológica de poeiras domiciliares na cidade de São Paulo. XIX Cong Bras de Alerg e Imunopatol e IV Cong Luso-brasil de Alergia e Imunopatol, Olinda, PE, p. 60.

Korsgaard J, Hallas TE 1979. Tarsonemid mites in Danish house dust. Allergy 34: 225-32.

Madeira NG, Sogayar MI 1993. Prevalência de Demodex folliculorum e Demodex brevis em amostra populacional de Botucatu, São Paulo, Brasil. Rev Soc Bras Med Trop 26: 221-224.

Mazzuco RM, Lebarbenchon MA, Moecke EHS, Meyer E, Spíndola-Batti MA, Fröde T, Morato EF 2000. Anafilaxia por ingestão de ácaros de estocagem. Rev Bras Alerg Imunopatol 23: 189.

Medeiros Jr M, Figueiredo JP 1997. Sensibilização a aeroalérgenos em indivíduos com asma brônquica e/ ou rinite crônica em Salvador, Bahia. Rev Bras Alerg Imunopatol 20: 143-154.

Mello JF, Baggio D, Zuppi L, Bellesi N, Croce J 1988. Ácaros do pó domiciliar de Belém, Icoraracy e Castanhal, no Pará. Rev Bras Alerg Imunopatol 11: A125.

Mendes E 1989. Alergia no Brasil: Alérgenos Regionais e Imunoterapia, Manole, São Paulo, 221 pp.

Moreira NS 1975. Acarinos Pyroglyphidae e outros Sarcoptiformes em Amostras de Pó Domiciliar em Belo Horizonte, Minas Gerais, MSc Thesis, Instituto de Ciências Biológicas, Universidade Federal de Minas Gerais.

Moreira NS 1978. Redescrição de Chortoglyphus arcuatus (Troupeau, 1878) (Sarcoptiformes, Chotoglyphidae). Rev Bras Biol 38: 245-249.

Moreira NS 1980. Alguns dados sobre ácaros de poeira doméstica de Belo Horizonte. Lundiana 1: 49-50.

Mori JC, Mello LM, Jardim RF, Aun WT, Mello JF 1993. Alérgenos e análise crítica do controle ambiental. Rev Bras Alerg Imunopatol 16: 174-180.

Muniz JRO, Pinho Jr AJ, Oliveira CH, Prado AP, Graudenz GS, Gonçalves B, Lazzarini S 1996. Ácaros em roupas: fonte de exposição antigência. Rev Bras Alerg Imunopatol 19: A152.

Naspitz CK, Diniz C, Rizzo MC, Fernández-Caldas E, Solé D 1997. Human scalps as a reservoir of domestic mites. Lancet 349: 404.

Oliveira CH 1999. Avaliação da Fauna Acarina em Amostras de Poeira de Colchões na Cidade de 
Campinas e Comparação com a Sensibilidade Cutânea Imediata de Pacientes Atópicos, MSc Thesis, Unicamp, Campinas, SP.

Oliveira CH, Graudenz GS, Gonçalves B, Pinho Jr AJ, Lazzarini S 1996. Prevalência de sensibilização em atópicos em Campinas/SP. Rev Bras Alerg Imunopatol 19: A49.

Oliveira RSB, Oliveira CH, Muniz JRO, Lazzarini S, Prado AP 1998a. Ácaros da poeira domiciliar na cidade de Campinas - Resultados preliminares. Rev Bras Alerg Imunopatol 21: A090.

Oliveira RSB, Oliveira CH, Graudenz GS, Barros PMG, Prado AP 1998b. Levantamento da fauna acarina em poeira do prédio do arquivo central da UNICAMP. Rev Bras Alerg Imunopatol 21: A091.

Oliveira RSB, Muniz JRO, Oliveira CH, Prado AP 1998c. Levantamento de ácaros em vestimentas de pacientes alérgicos à poeira domiciliar. III CAEB - Cong Aberto aos Estudantes de Biologia, Unicamp, Campinas, SP, p S1.51.

Oliveira CH, Binotti RS, Muniz JRO, Pinho Jr AJ, Prado AP, Lazzarini S 1999. Fauna acarina da poeira de colchões na cidade de Campinas-SP. Rev Bras Alerg Imunopatol 22: 188-197.

Platts-Mills TAE, Chapman MD 1987. Dust mites: immunology, allergic disease, and environmental control. J Allergy Clin Immunol 90: 755-775.

Rizzo MC, Arruda LK, Chapman MD, FernandezCaldas E, Baggio D, Platts-Mills TAE, Naspitz CK 1993. IgG and IgE antibody responses to dust mite allergens among children with asthma in Brazil. Ann Allergy 71: 152-158.

Romano NS, Baggio D, Carvalho JPP, Flechtmann CHW 1987. Ácaros contaminantes de ambiente, encontrados em doces derivados do leite e do chocolate, a venda em pequenos estabelecimentos da cidade de São Paulo, SP. Resumos do XI Cong Brasil de Entomologia, Vol 1, Campinas, SP, p. 24.

Rosa AE 1978. Estudo sobre a Fauna Acarina em Poeira
Doméstica no Brasil, MSc Thesis, Escola Superior de Agricultura 'Luiz de Queirós', Universidade de São Paulo.

Rosa AE, Flechtmann CHW 1979. Mites in house dust from Brazil. Intl J Acar 5: 195-198.

Rosário Filho NA, Baggio D, Suzuki MM, Thomaz PCP, Sugisawa S, Hanggi V 1992. Ácaros na poeira domiciliar em Curitiba. Rev Bras Alerg Imunopatol 15: A25.

Sampaio FAA, Rocha YV 1985. Levantamento da acarofauna de poeira domiciliar em Campinas (SP) com especial ênfase nas famílias Pyroglyphidae e Glycyphagidae. Resumos do XII Cong Brasil de Zoologia, Campinas, SP, p. A84.

Sarinho ESC, Coutinho VB, Robalinho TI, Coutinho HB, Solé D, Carvalho B 1994a. Dermatophagoides farinae em residências de atópicos do Recife - Estudo descritivo. Rev Bras Alerg Imunopatol 17: A63.

Sarinho ESC, Coutinho VB, Robalinho TI, Coutinho HB, Solé D, Carvalho B 1994b. Identificação de ácaros em residências de alérgicos no Recife - relato de casos. Rev Bras Alerg Imunopatol 17: A64.

Serravale K, Medeiros Jr M 1998. Fauna acarina na cidade de Salvador, Bahia. Rev Bras Alerg Imunopatol 21: A093.

Serravalle K, Medeiros Jr M 1999. Ácaros da poeira domiciliar na cidade de Salvador, BA. Rev Bras Alerg Imunopatol 22: 19-24.

Silva DAO, Sopelete MC, Souza GG, Arruda-Chaves E, Arruda LK, Chapman MD, Taketomi EA 1999. Indoor allergen exposure among asthmatic patients living in Uberlândia, MG, Brazil. J Allergy Clin Immunol 103: S187.

Spalding SM, Bernd LAG, Wald V, Barbiero JP, Ambrózio LC 1997. Serum specific IgE and skin prick test reactivity to $D$. pteronyssinus and B. tropicalis: a controlled study. J Allergy Clin Immunol 99: S345.

Wharton GW 1976. House dust mites - Review article. $J$ Med Entomol 12: 577-621. 